COMMENT. The clinical and MRI findings are usually sufficient evidence on which to base a decision to offer resective surgery in the majority of children with medically intractable epilepsy. Ictal EEG video recordings provide confirmatory evidence of focal lesions, but in situations with limited resources, they may be reserved for children with bilateral MRI changes or normal MRI. SPECT findings do not influence a decision to operate. The role of ictal EEG in estimating prognosis following surgery requires further study. Psychological assessment is an additional factor in the decision making process for epilepsy surgery and in the evaluation of its benefits.

Extratemporal ictal clinical features in hippocampal sclerosis are most frequent in cases with severe hippocampal atrophy but do not affect surgical outcome, in a study at the University of Verona, Italy; and National Hospital, London, UK. (Borelli P, Shorvon SD, Stevens JM, et al. Epilepsia Aug 2008;49:1333-1339).

\title{
SPIKE FREQUENCY AND SURGICAL OUTCOME IN TLE WITH UNILATERAL HIPPOCAMPAL ATROPHY
}

Prognostic implications of absolute spike frequency over the affected temporal lobe and relative spike distribution between the two temporal lobes for postoperative seizure control were assessed in 55 adult patients with medically refractory mesial temporal lobe epilepsy (MTLE), in a study at General Hospital Hietzing, Vienna, Austria. Age at seizure onset was $11.2+/-9.3$ years, and age at evaluation was $34.7+/-8.1$ years. Presurgical evaluation included prolonged video-EEG monitoring. One year following surgery, 4 of 14 patients $(28.6 \%)$ in the frequent spike group $(>60 / \mathrm{hr}$ over the affected temporal lobe) were completely seizure free compared to 33 of 41 patients $(80.5 \%)$ in the nonfrequent spikes group $(<60 / \mathrm{hr})(\mathrm{p}=0.001)$. In the unitemporal group $(>90 \%$ spikes over the affected temporal lobe), 25 of 35 patients $(71 \%)$ were completely seizure free compared to 12 of 20 patients $(60 \%)$ in the bitemporal spike group $(\mathrm{p}=0.282)$. Relative spike distribution over the two temporal lobes was not significantly related to postoperative outcome. (Krendl R, Lurger S, Baumgartner C. Absolute spike frequency predicts surgical outcome in TLE with unilateral hippocampal atrophy. Neurology Aug 2008;71:413-418). (Respond: Dr C Baumgartner. Email: christoph baumgartner( $\iota$ wienkav.at).

COMMENT. Absolute spike frequency over the affected temporal lobe, but not relative spike distribution between the two temporal lobes, is a strong predictor of postoperative seizure control in young adults with medically refractory mesial temporal lobe epilepsy. TLE patients with a low spike frequency over a unilateral hippocampal atrophy should be excellent candidates for epilepsy surgery.

\section{NOVEL LOCUS FOR GENERALIZED EPILEPSY WITH FEBRILE SEIZURES PLUS}

Linkage analysis was conducted in 5 French families with generalized epilepsy with febrile seizures plus (GEFS+), with at least 7 affected members with autosomal dominant transmission, in an attempt to localize a new gene for GEFS+, at Hospitalier Pitie-Salpetriere, 